\title{
BALAYAGE DEFINED BY THE NONNEGATIVE CONVEX FUNCTIONS ${ }^{1}$
}

\author{
P. FISCHER AND J. A. R. HOLBROOK
}

Abstract. We study the Choquet order induced on measures on a linear space by the cone of nonnegative convex functions. We are concerned mainly with discrete measures, and the following result is typical. Let $x_{1}, \ldots, x_{r}, y_{1}, \ldots, y_{n}$, where $r<n$, be points in $\mathbf{R}^{d}$. Then

$$
\sum_{1}^{r} f\left(x_{k}\right)<\sum_{1}^{n} f\left(y_{k}\right)
$$

for all nonnegative, continuous, convex functions $f$ if, and only if, there exists a doubly stochastic matrix $M$ such that

$$
x_{j}=\sum_{k=1}^{n} m_{j k} y_{k} \quad(j=1, \ldots, r) .
$$

In the case $d=1$, this result may be found in the work of $L$. Mirsky; our methods allow us to place such results in a general setting.

1. The key step. Here we deal with Baire measures (always nonnegative and finite) on a compact subset $K$ of a Hausdorff space $E$. We shall write $\mu(f)$ for the integral of a continuous (real-valued) function $f$ on $K$ with respect to the measure $\mu$, and $\delta_{x}$ for the unit mass at $x \in K$. When $K$ has a convex structure, we write $C$ for the cone of continuous convex functions on $K$ and $C^{+}$for the nonnegative members of $C$.

If $\mu(f)<\nu(f)$ for all $f \in C^{+}$, we follow a common terminology (see, e.g., P. A. Meyer [3, Chapter XI, §3]) in saying that the measure $\nu$ is a "balayage" of $\mu$ relative to the class $C^{+}$. The following theorem relates balayage relative to $C^{+}$to balayage relative to $C$.

TheOREM 1. Let $\mu, \nu$ be two Baire measures on a compact convex subset $K$ of $a$ locally convex topological vector space $E$. If $\nu$ is a balayage of $\mu$ relative to $C^{+}$, then there exists a Baire measure $\lambda$ such that $\nu$ is a balayage of $\mu+\lambda$ relative to $C$. Furthermore we may choose $\lambda$ to be concentrated at a point $x_{0} \in K$ if we wish: $\lambda=(\nu(1)-\mu(1)) \delta_{x_{0}}$.

Proof. By the Riesz representation theorem, each (nonnegative) Baire measure $\lambda$ corresponds to a continuous linear functional $\phi$ on $C(K)$ such that $\phi(f)>0$ for all $f$ in the (convex) cone $P$ of positive functions in $C(K)$. If we find such a $\phi$ with the additional properties

Received by the editors July 26, 1978 and, in revised form, July 27, 1979.

1980 Mathematics Subject Classification. Primary 26B25, 26D15, Secondary 15A39, $15 A 51$.

Key words and phrases. Hardy-Littlewood-Pólya order, doubly stochastic matrices, balayage.

${ }^{1}$ Work supported in part by the National Research Council of Canada under grant A-8745. 


$$
\phi(f)<(\nu-\mu)(f) \quad\left(f \in C^{+}\right)
$$

and

$$
\phi(1)=(\nu-\mu)(1),
$$

then $\nu$ is a balayage of $\mu+\lambda$ relative to $C$, since $C=\left\{f+r 1: f \in C^{+}, r \in \mathbf{R}\right\}$.

If $\mu(1)=\nu(1)$ we can simply put $\phi=0$. Otherwise, setting $\alpha=\nu-\mu$, we have $\alpha(1)>0$ and we can define the linear operator $T$ on $C(K)$ by

$$
T f=f-(\alpha(f) / \alpha(1)) 1 .
$$

Now $f \in C^{+}$implies $T f \in C$ and, we claim, $T f \notin P$; otherwise we would have $0<m=\min T f$, and $T f-m 1 \in C^{+}$so that

$$
0<\alpha(T f-m 1)=\alpha(f-(\alpha(f) / \alpha(1)) 1)-\alpha(m 1)=-m \alpha(1)<0,
$$

a contradiction. Thus the cone $\mathrm{TC}^{+}$and the open cone $P$ do not intersect, so that by a well-known form of the separation theorem (see, e.g., W. Rudin [6, Theorem 3.4]) there is a continuous linear functional $\psi \neq 0$ on $C(K)$ such that $\psi(f)<\psi(g)$ whenever $f \in T C^{+}$and $g \in P$.

Since $P$ is an open cone and $\psi \neq 0$, it is clear that $\psi(P)$, being bounded below, must be $(0, \infty)$. If we set $\phi=(\alpha(1) / \psi(1)) \psi$, then $\phi(1)=\alpha(1)$, and it remains to show that $\phi(f)<\alpha(f)$ whenever $f \in C^{+}$. But in this case we have ensured that $\phi(T f)<0$, so that $\phi(f)<\phi((\alpha(f) / \alpha(1)) 1)=\alpha(f)$.

Finally we note that we can, if we wish, replace $\lambda$ by its resultant $\lambda(1) \delta_{x_{0}}$; more precisely, let $x_{0}$ be the barycentre of the probability measure $\lambda / \lambda(1)$ (see $R$. R. Phelps [5, Proposition 1.1]). By definition, $x_{0}$ is that point in $K$ such that $\delta_{x_{0}}(f)$ $\left(=f\left(x_{0}\right)\right)=\lambda(f) / \lambda(1)$ for all continuous affine functions $f$ on $K$, and it is well known that the inequality $\lambda(1) f\left(x_{0}\right)<\lambda(f)$ follows for all $f \in C$. Thus we may replace $\lambda$ by $\lambda(1) \delta_{x_{0}}=(\nu(1)-\mu(1)) \delta_{x_{0}}$. Q.E.D.

2. Application. As we shall make clear in the remarks below, the following theorem provides a common extension of some basic results on balayage for discrete measures.

THEOREM 2. Let $x=\left(x_{1}, \ldots, x_{r}\right), y=\left(y_{1}, \ldots, y_{n}\right)$ where $r<n$ and $x_{k}, y_{k}$ are elements of $\mathbf{R}^{d}$. Then the following are equivalent.

(i) $\Sigma_{1}^{r} f\left(x_{k}\right)<\Sigma_{1}^{n} f\left(y_{k}\right)$ for every convex continuous function $f: K \rightarrow \mathbf{R}^{+}$, where $K$ is the convex hull in $\mathbf{R}^{d}$ of the $x$ 's and $y$ 's.

(ii) $x=[M y]_{r}$ for some doubly stochastic matrix $M$ (here $[z]_{r}$ denotes the vector formed by the first $r$ components of the vector $z$ and the product $M y$ is interpreted formally with $y$ as a column vector).

Remarks. (a) In the one-dimensional case $(d=1)$ this result comes from $\mathrm{Ch}$. Davis and L. Mirsky (see [4]). In that case, the well-known relationship between doubly stochastic matrices and the Hardy-Littlewood-Pólya order allows the addition of a third equivalent statement:

(iii) $x=[z]$, for some $z \in \mathbf{R}^{n}$ such that

$$
\sum_{k=1}^{i} z_{k}^{*}<\sum_{k=1}^{i} y_{k}^{*}
$$


for $i=1, \ldots, n$ with equality holding for $i=n$, where $z^{*}$ and $y^{*}$ denote the nonincreasing rearrangements of $z$ and $y$.

(b) The general $d$-dimensional form of Theorem 2 is due to S. Sherman [7] and C. Stein (see D. Blackwell [1]) in the special case $r=n$. Note that in this case there is no need to require nonnegative functions in (i) since the inequality is unchanged upon adding any constant to $f$. We shall prove Theorem 2 by deriving it from the special case of Sherman and Stein.

Proof. (i) $\Rightarrow$ (ii). In the terminology of Theorem 1 , the hypothesis says that $\nu=\Sigma_{1}^{n} \delta_{y_{k}}$ is a balayage of $\mu=\Sigma_{1}^{r} \delta_{x_{k}}$ relative to $C^{+}$. By Theorem 1 , there exists $x_{0}$ such that $\nu$ is a balayage of $\mu+(n-r) \delta_{x_{0}}$ relative to $C$. Thus $z=$ $\left(x_{1}, \ldots, x_{r}, x_{0}, \ldots, x_{0}\right)$ and $y$ satisfy the hypothesis (i) of the Sherman-Stein theorem, so that $z=M y$ for some doubly stochastic $M$. Hence $x=[z]_{r}=[M y]_{r}$.

(ii) $\Rightarrow$ (i). By the theorem of Sherman and Stein and the nonnegativity of $f$, we have, with $z=M y$,

$$
\sum_{1}^{n} f\left(y_{k}\right)>\sum_{1}^{n} f\left(z_{k}\right)>\sum_{1}^{r} f\left(z_{k}\right)=\sum_{1}^{r} f\left(x_{k}\right) .
$$

Q.E.D.

3. A variant. In [2] an analogue of the Sherman-Stein theorem is established for substochastic matrices. One form of this result may be stated as follows (cf. [2, Théorème 8]).

THEOREM 3. Let $x=\left(x_{1}, \ldots, x_{n}\right), y=\left(y_{1}, \ldots, y_{n}\right)$ where $x_{k}$ and $y_{k}$ are elements of $\mathbf{R}^{d}$. Then the following statements are equivalent.

(i) $\sum_{k=1}^{n} f\left(x_{k}\right)<\sum_{k=1}^{n} f\left(y_{k}\right)$ for every continuous convex function $f: \mathbf{R}^{d} \rightarrow \mathbf{R}$ such that $f>f(0)$.

(ii) there exists an $n \times n$ doubly substochastic matrix $M$ such that $x=M y$.

The following theorem extends Theorem 3 in the same way that Theorem 2 extends the Sherman-Stein theorem.

THEOREM 4. Let $x=\left(x_{1}, \ldots, x_{r}\right), y=\left(y_{1}, \ldots, y_{n}\right)$, where $r<n$ and the $x_{k}$ and $y_{k}$ are elements of $\mathbf{R}^{d}$. Then the following statements are equivalent.

(i) $\sum_{k=1}^{r} f\left(x_{k}\right)<\sum_{k=1}^{n} f\left(y_{k}\right)$ for every continuous convex function $f: \mathbf{R}^{d} \rightarrow \mathbf{R}^{+}$such that $f>f(0)$.

(ii) there exists an $n \times n$ doubly substochastic matrix $M$ such that $x=[M y]_{r}$.

Proof. (ii) $\Rightarrow$ (i). This follows by an obvious modification of our proof of the corresponding implication in Theorem 2.

(i) $\Rightarrow$ (ii). For any convex continuous $f: \mathbf{R}^{d} \rightarrow \mathbf{R}$ such that $f>f(0)$ the function $f-f(0)$ satisfies the hypotheses and the resulting inequality clearly implies that

$$
\sum_{1}^{r} f\left(x_{1}\right)+(n-r) f(0)<\sum_{1}^{n} f\left(y_{k}\right) \text {. }
$$

Using the implication (i) $\Rightarrow$ (ii) of Theorem 3, we see that there exists a doubly substochastic matrix $M$ so that $\left(x_{1}, \ldots, x_{r}, 0, \ldots, 0\right)=M\left(y_{1}, \ldots, y_{n}\right)$. Q.E.D. 


\section{REFERENCES}

1. D. Blackwell, Equivalent comparisons of experiments, Ann. Math. Statistics 24 (1953), 265-272.

2. P. Fischer and J. A. R. Holbrook, Matrices sous-stochastiques et fonctions convexes, Canad. J. Math. 29 (1977), 631-637.

3. P. A. Meyer, Probability and potentials, Blaisdell, Waltham, Mass., 1966.

4. L. Mirsky, Majorization of vectors and inequalities for convex functions, Monatsh. Math. 65 (1961), 159-169.

5. R. R. Phelps, Lectures on Choquet's theorem, Van Nostrand, Princeton, N. J., 1966.

6. W. Rudin, Functional analysis, McGraw-Hill, New York, 1973.

7. S. Sherman, On a theorem of Hardy, Littlewood, Pólya and Blackwell, Proc. Nat. Acad. Sci. U. S. A. 37 (1951), 826-831.

Departignt of Mathematics and Statistics, University of Gublph, Gubluph, Ontaruo, Canada 